[9] J. Canny, "A computational approach to edge detection," IEEE Trans. Pattern Anal. Machine Intell., vol. PAMI-8, June 1986.

[10] G. Courbebaisse and C. Ducottet, "Time frequency and time scale distributions applied to the measurement of turbulent structures," ASMEFED, vol. 239 , no. 4 , pp. 181-188, 1996

[11] F. Attneave, "Some informational aspects of visual perception," Psychol. Rev., vol. 61, no. 3, pp. 183-193, 1954

[12] P. V. Sankar and C. V. Sharma, "A parallel procedure for the detection of dominant points on a digital close curves," Comput. Graph. Image Process., vol. 7, pp. 403-412, 1978.

[13] J. Fayolle, C. Ducottet, T. Fournel, and J. P. Schon, "Motion characterization of unrigid objects by detecting and tracking feature points," in Proc. IEEE ICIP, 1996, vol. 3, pp. 803-806.

[14] J. Fayolle, "Study of image processing techniques for motion determination for unrigid objects, application to the measurement of displacement in flows," Ph.D. dissertation, Univ. Saint Etienne, Saint Etienne, France, 1996.

\section{A Robust Technique for Image Descreening Based on the Wavelet Transform}

\author{
Jiebo Luo, Ricardo de Queiroz, and Zhigang Fan
}

\begin{abstract}
In this correspondence, a novel wavelet-based approach to recover continuous-tone (contone) images from halftone images is presented. Wavelet decomposition of the halftone image facilitates a series of spatial and frequency selective processing to preserve most of the original image contents while eliminating the halftone noise. Furthermore, optional nonlinear filtering can be applied as a postprocessing stage to create the final aesthetic contone image. This approach lends itself to practical applications since it is independent of parameter estimation and, hence, universal to all types of halftoned images, including those obtained by scanning printed halftones.
\end{abstract}

\section{INTRODUCTION}

Halftoning is a quantization process that reduces the bit-depth of a digital image while trying to maintain its gray-scale appearance [1]. The main applications of halftoning are printing and displaying, where continuous-tone (contone) pictures are reproduced on binary output devices. Descreening, or retrieving a contone image from a halftone, is a problem not only of great theoretical interest as an illposed inverse problem but also of significant practical usage. Halftone images are usually hostile to many common image processing procedures, such as compression, scaling, rotation, rescreening, or tone correction. As a result, descreening is usually the first step for halftone image processing.

Most digital halftoning methods [1] can be classified into three categories: ordered dithering, error diffusion, and optimization-based methods. Error diffusion and optimization-based methods usually produce halftones of scattered pixels, whereas ordered dithering can

Manuscript received February 15, 1997; revised November 30, 1997. The associate editor coordinating the review of this paper and approving it for publication was Prof. P. P. Vaidyanathan.

J. Luo was with the Digital Imaging Technology Center, Xerox Corp. Webster, NY 14580 USA. He is now with the Imaging Science Technology Laboratory, Eastman Kodak Co., Rochester, NY 14650 USA (e-mail: luo@image.kodak.com).

R. de Queiroz and Z. Fan are with the Digital Imaging Technology Center, Xerox Corp., Webster, NY 14580 USA (e-mail: queiroz@wrc.xerox.com; Zhigang_Fan@wb.xerox.com).

Publisher Item Identifier S 1053-587X(98)02608-7. generate both clustered dots or dispersed dots. The spectrum of the halftoning noise is largely highpass in nature. Nevertheless, it differs significantly for various processes. The noise energy of clustered dot screening is concentrated in a few spikes at screen frequencies and their harmonics [2]. On the other hand, the noise spectra of other halftone techniques, such as error diffusion and optimization methods, are usually smooth and continuous. The statistics of images differ significantly between orthographical halftones and scanned ones. The former refer to the halftones created digitally and acquired in a digital form, whereas the latter refer to the halftones printed and then obtained through a scanner. The descreening of scanned halftones is particularly useful for many practical applications. As a first-order approximation, the scanned halftone can be considered a lowpass filtered version of the original halftone. In orthographic halftones, the value (black or white) of each pixel reveals a possible range of the input value (or modified input value for the error diffusion) of the pixel. This provides important clues for descreening. However, this benefit usually does not exist for scanned halftones because they lack the perfect registration typical of orthographic ones.

Since halftoning noise is mainly concentrated in high frequency, it can be effectively removed by lowpass filtering, which, however, tends to blur edges and destroy fine details in the original image. Many different methods were reported to improve descreening [3]-[10]. They can be roughly divided into two groups. The first kind of approach is to treat descreening as a constrained optimization problem [3]-[7]. Perfect registration and availability of halftoning parameters are assumed. The possible range of input (or modified input for error diffusion) is then utilized as a constraint. The second class of method is based on edge-preserving smoothing [5], [8], [9], which attempts to remove a screen without blurring details. There are also algorithms combining smoothing approaches and constrained optimization approaches [10]. Most of the descreening methods are designed for a particular halftoning process. Some of them are tuned or trained for a particular set of halftone parameters. They fail or perform poorly when the condition changes. They also often assume a priori knowledge of halftoning parameters, such as screening frequencies, threshold matrices, or error diffusion weights. However, in real applications, the original halftoning methods and their associated parameters are generally unknown. A robust estimation is usually difficult to achieve. Moreover, the image is often obtained by scanning halftone materials.

Our novel descreening approach is facilitated by the wavelet transform or by overcomplete subband representations. It is independent of the halftoning method and is applicable to scanned halftones.

\section{BAsic Descreening Methodology}

We propose a descreening scheme composed of sequential stages:

i) subband decomposition;

ii) noise attenuation in nonedge areas;

iii) oriented filtering;

iv) inverse subband recomposition;

v) an optional nonlinear edge-preserving postfiltering stage.

The halftone image is first decomposed into different frequency subbands using the DWT with separable filter banks [11], as in Fig. 1. Although the energy of the halftoning process resides primarily in the high-frequency subbands, the low-frequency subbands contain most of the signal energy. The high-frequency subbands, where the signal energy and halftoning energy are heavily mixed together, contain signal energy corresponding to edge information. It is important to 

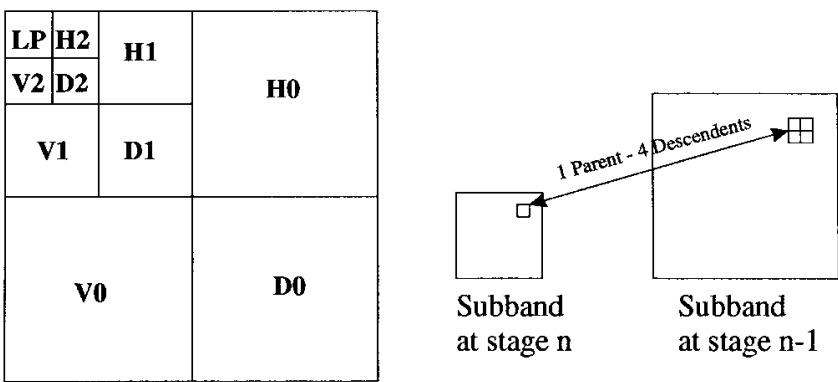

Fig. 1. DWT decomposition and its parent-descendent relationship.

differentiate high-frequency image contents from the halftone noise [2]. In the past, frequency domain-oriented descreening approaches, i.e., lowpass filtering-based approaches, have not been very successful due to the heavy mixture of halftone noises with high-frequency image contents such as edges and texture. While an increasing amount of halftone noise can be removed by applying lowpass filtering with a lower cut-off frequency, the increasing amount of loss of highfrequency image contents inevitably causes more severe degradation of the reconstructed contone image.

Wavelet decomposition facilitates selective processing in both the frequency and space domains. After the wavelet decomposition, a joint space-frequency representation is obtained. There are several properties of such a representation that are significant and will be exploited by the proposed descreening approach. First, each high-frequency subband image has a distinctive orientation. Second, each high-frequency subband image is composed of coefficients corresponding to similar frequency components at different locations. Third, each high-frequency subband coefficient is part of a hierarchical tree composed of coefficients corresponding to different frequency components in the same spatial neighborhood.

\section{A. Noise Attenuation (Frequency Correlation)}

There exists a hierarchical relationship among the amplitudes of the subband samples at different resolution levels but the same spatial location. For a given coefficient (parent), there are four coefficients (children) at the same spatial location in the subband of the same orientation at the next finer resolution. This relationship is illustrated in Fig. 1. It is found that in general, the magnitudes of the descendants are nonincreasing with respect to their ancestors. In other words, the power spectrum of the image signal commonly decays as the frequency increases. This assumption has been successfully applied to image coding [12]. The violation of this constraint is often an indication of the presence of halftoning energy. Therefore, we clip the coefficients in the high-frequency subbands so that their magnitudes are not larger than the magnitude or weighted magnitude of their parents. We refer to this process as clipping, which, unlike lowpass filtering, is spatially adaptive.

Clipping weights may vary from level to level, and the clipping process can be made more spatially adaptive (conservative over edges and aggressive otherwise). The weights have the primary responsibility to equalize the gain of the subbands. If orthonormal or near-orthonormal bases are used, a gain factor of 2 is applied to the dynamic range of subbands each time a subband is further decomposed (in order to ensure the energy conservation). Apart from the simple renormalization task, the weights can be used to clip aggressively high-frequency subbands (using smaller weights) and to clip conservatively low-frequency subbands (using larger weights). In high-frequency bands, the original image energy is small, and its features are less visible. On the other hand, low-frequency bands have less halftone energy mixed with important image features. Thus, being
TABLE I

Filter Kernels for Intraband ORIented Filtering, Where $a_{1}=\alpha, b_{1}=(1-\alpha) / 2, a_{2}=3 \alpha / 5, b_{2}=(5-3 \alpha) / 20$, AND $\alpha$ Is a Bandwidth Control Parameter For the Filters

\begin{tabular}{|c|c|c|}
\hline horizontal & vertical & diagonal \\
\hline $0 b_{1} 0$ & $\begin{array}{lll}0 & 0 & 0\end{array}$ & $b_{2} \quad 0 \quad b_{2}$ \\
\hline $0 a_{1} 0$ & $a_{1} a_{1} b_{1}$ & $a_{2} 0$ \\
\hline $\begin{array}{lll}0 & b_{1} & 0\end{array}$ & $\begin{array}{llll}0 & 0 & 0\end{array}$ & $b_{2} 0 b_{2}$ \\
\hline
\end{tabular}

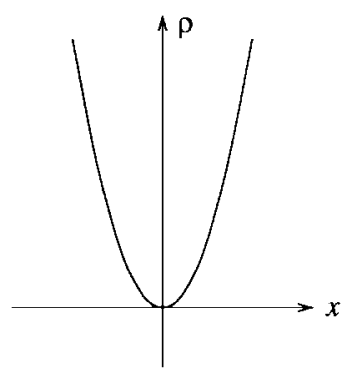

$\operatorname{GMRF}(\mathrm{p}=2)$

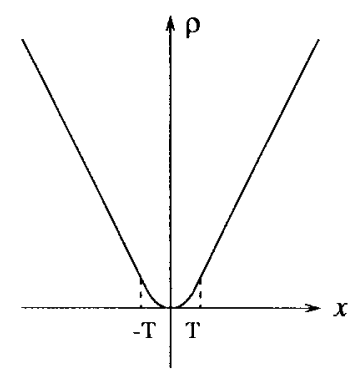

HMRF $(T=1)$
Fig. 2. Quadratic potential function and the Huber minimax potential function.

conservative when clipping lower frequency subbands is advisable, but weight selection has to be made after extensive empirical tests.

Halftone patterns are not well correlated among different frequency bands at same spatial position. The same is not true for prominent edges in the image, which may show up across all subbands at the same orientation and location. Wherever there is activity in a parent coefficient, one should be more conservative and give larger weights for the parent before clipping. On the other hand, low activity in the parent coefficient region may demand a more aggressive clipping (lower weights) to ensure that halftone noise is not reproduced in smooth areas of the image. Therefore, adaptive clipping may come in the form of space-varying weight distribution aimed at improving halftone noise removal while preserving edges.

\section{B. Intraband Filtering (Spatial Correlation)}

In order to further reduce the halftone noise near edges, one must first locate the edges. Fortunately, within the framework of wavelet decomposition, edge contents have been decomposed into wellseparated subband images with respect to frequency, location, and orientation. It is well known that each subband image contains edge components primarily along its orientation. The horizontal subband is obtained by lowpass filtering in the vertical direction and by highpass filtering in the horizontal direction. The opposite configuration yields the vertical subband, whereas the diagonal subband is a product of highpass filtering in both directions. As a result, vertical subbands contain horizontal edge components, horizontal subbands contain vertical edge components, and diagonal subbands contain residual components in the diagonal and off-diagonal directions. Therefore, it is intuitive that if we lowpass filter the subband images along specific orientations, we might remove noisy components and not degrade sharp edges extensively. In particular, a one-dimensional (1-D) lowpass filter can be applied to both horizontal and vertical subbands, i.e., a horizontal lowpass filter is applied to the vertical subband and vice-versa, whereas an X-shaped lowpass filter is applied to the diagonal subbands. For example, one can use the filter kernels shown in Table I. Alternatively, explicit edge estimation can also be implemented to guide the filtering. 

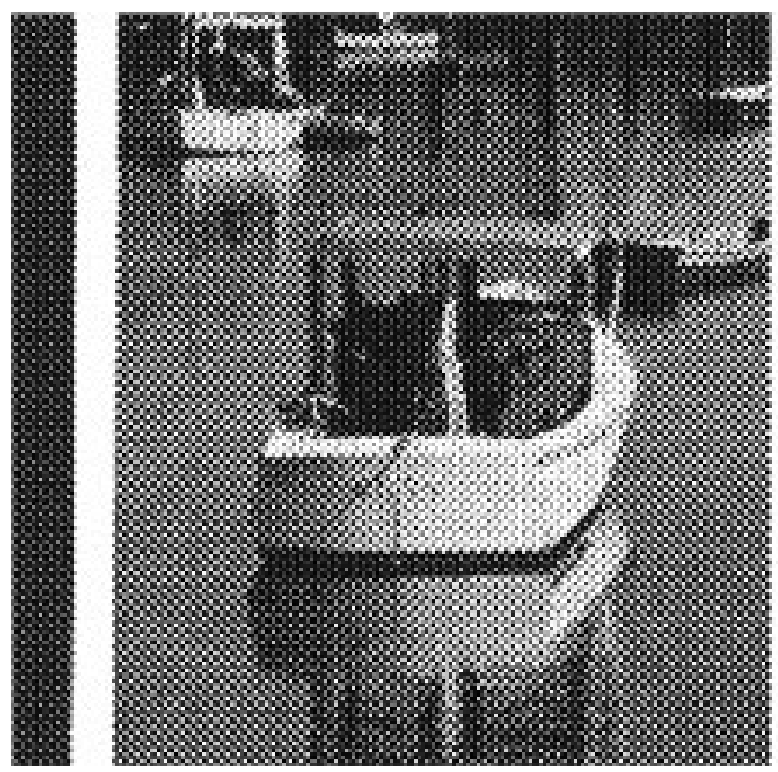

(a)

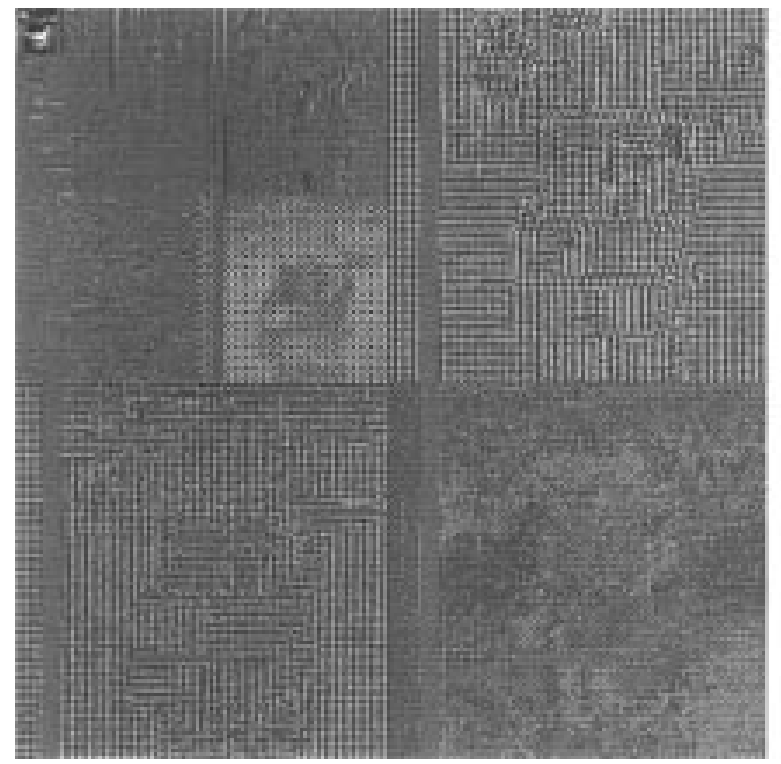

(c)
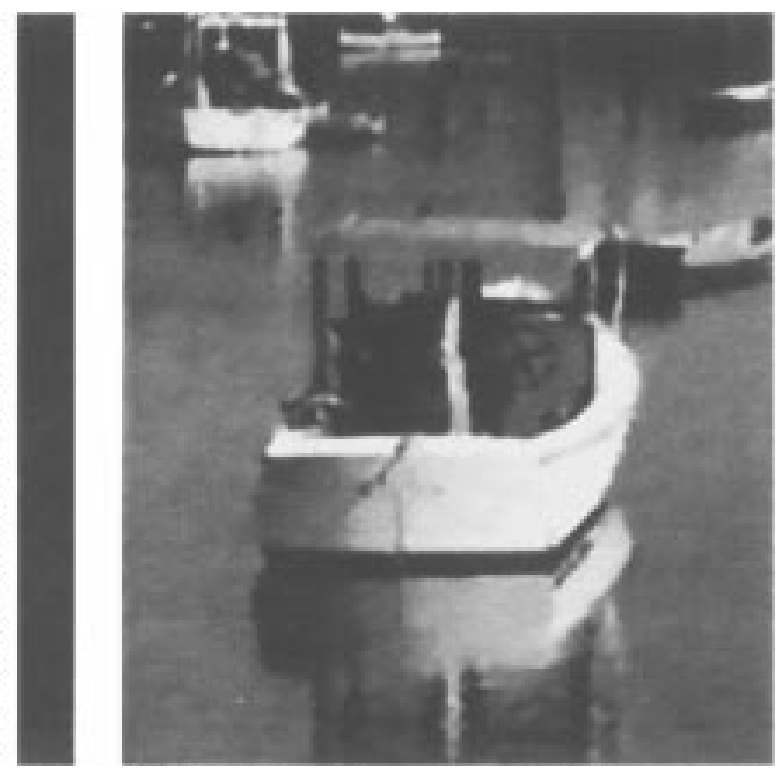

(b)

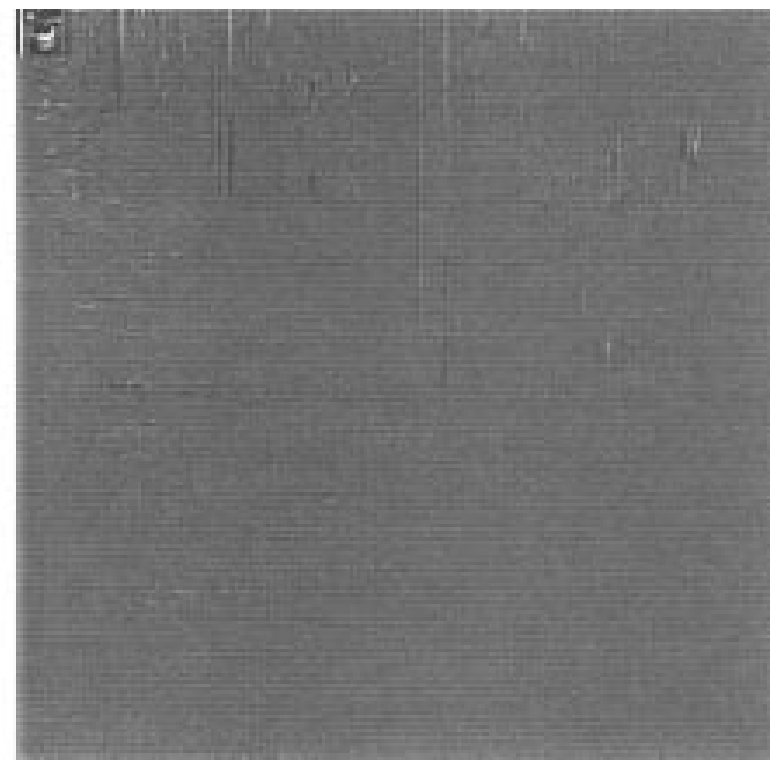

(d)

Fig. 3. (a) Halftone image and (b) its reconstructed contone version. Image size is $400 \times 400$, and the halftoning method employs $3 \times 6$ clustered dots. The four-level wavelet transform of the halftone image is shown in (c), and its processed (cleaned) subbands are shown in (d).

\section{Nonlinear Postfiltering}

The reconstructed contone image from inverse wavelet recomposition may still suffer from some artifacts, such as ringing and blotchiness. The most observable is the ringing artifact around prominent edges, which is frequently encountered in wavelet-based image processing. In this case, it is caused by partial loss of highfrequency components, although we are able to capture the most significant portion of the edge contents through the previous selective noise attenuation processes within the subbands.

The reconstructed contone image is preferably enhanced in the original space domain, using a nonlinear filtering with the spatial smoothness constraints modeled by a discontinuous image prior model, in particular, the Huber Markov random field (HMRF) [7], which has been used to various image smoothing applications [7], [13]. It is convenient to parameterize a (Markov) Gibbs random field (GRF) using a Gibbs potential function and a proper neighborhood system [13]. Convex potential functions with smooth transition, i.e., with good continuity properties, result in desired continuity in the image. However, there may be significant discontinuities such as edges in the image. As shown in Fig. 2, the quadratic Gaussian prior model (GPM), which corresponds to mean square error (MSE) criterion, is unable to differentiate these discontinuities or the outliers of the model, and its linear lowpass filtering nature tends to blur image edges and other details excessively and indiscriminately [13]. However, discontinuous (in terms of first or higher order derivatives) functions are more desirable because they are capable of being adaptive to discontinuities, e.g., the Huber minimax function shown in Fig. 2, where $T$ is a threshold, and $x$ denotes the difference between neighboring pixels. According to the difference of gray level between the current pixel and its neighbors, the quadratic segment of the function imposes least mean square smoothing of the artifacts when the local variation is below $T$, whereas the linear segment of the 

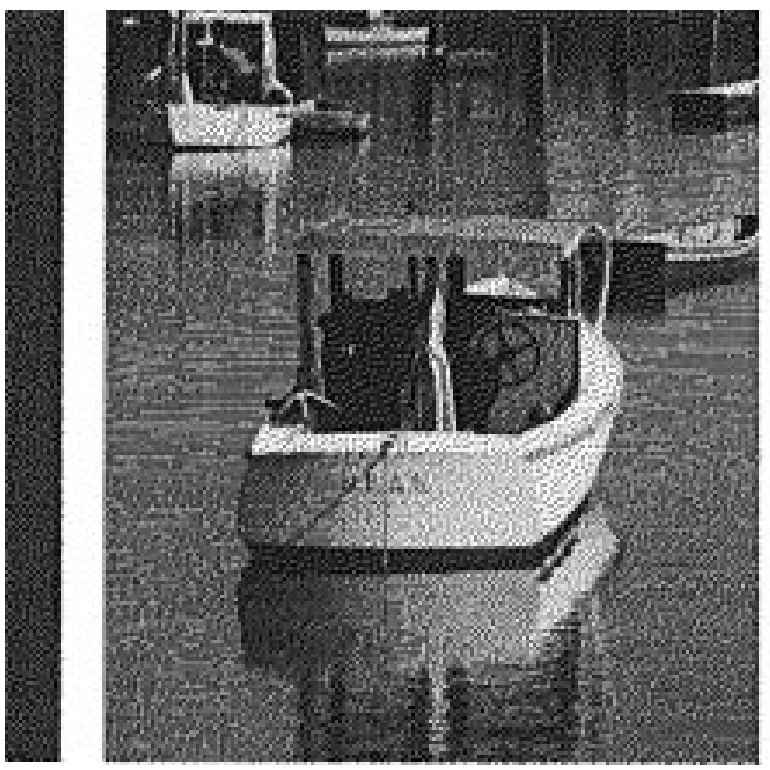

(a)
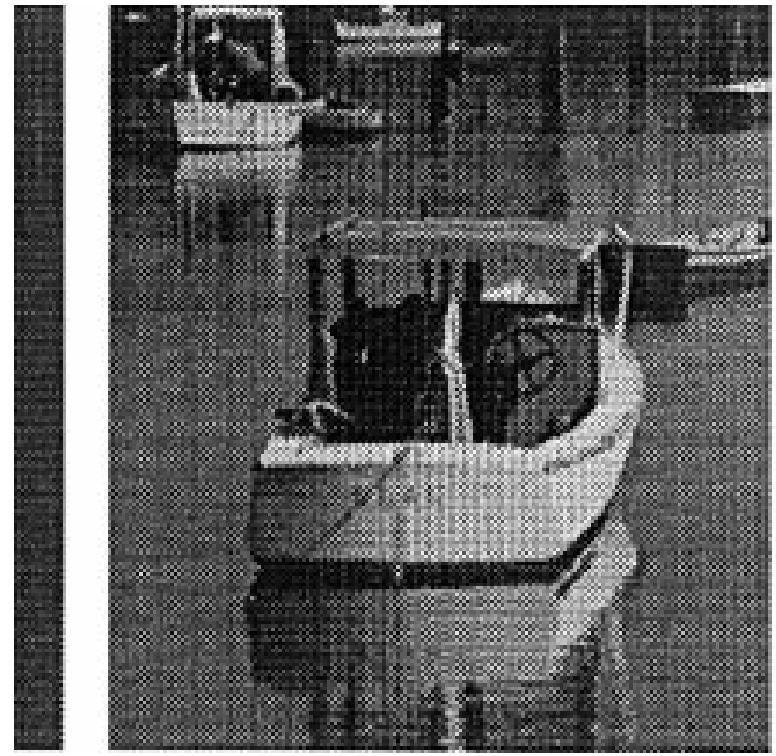

(c)

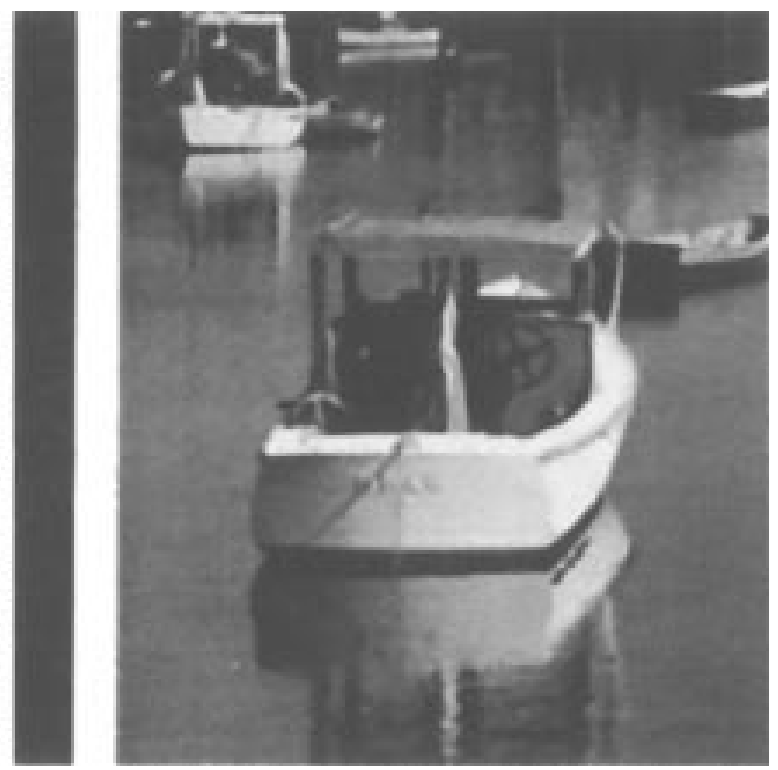

(b)

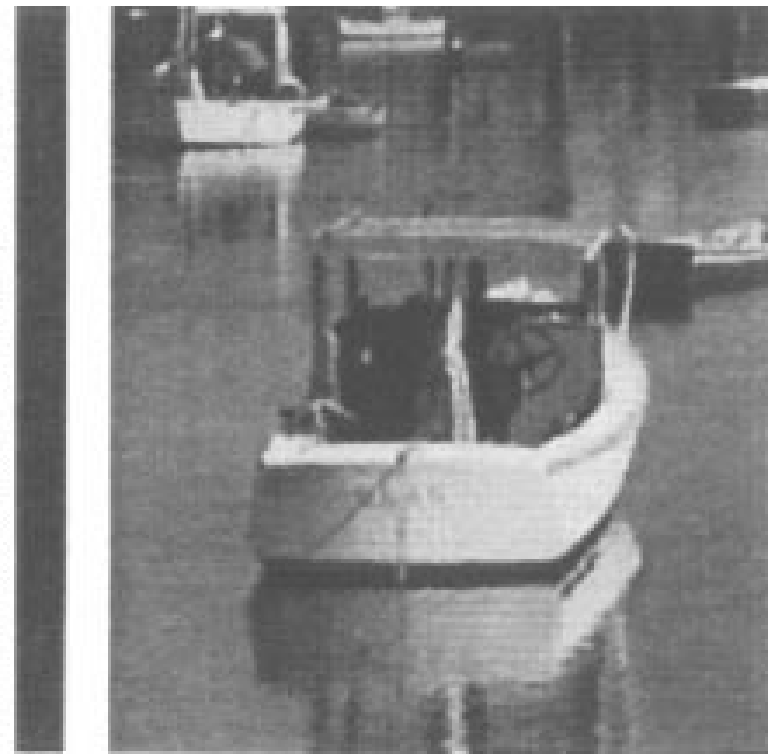

(d)

Fig. 4. Reconstructed contone versions from halftone images. (a) Error diffusion halftone. (b) Its reconstructed version. (c) Scanned halftone. (d) Its reconstructed version.

function enables the preservation of image details by allowing large discontinuities in the image with a much lighter penalty.

Other edge-preserving filtering methods (such as $\sigma$-filter [14] based filtering) are also applicable at this stage. A $\sigma$ filter behaves mostly like a linear convolution filter, except for just including samples whose levels are within $\pm \sigma$ levels from the center pixel. Thus, sharp edges are not blurred by the process. In practice, the best enhancement results are obtained by sequentially processing the reconstructed contone image with HMRF-based filtering and $\sigma$ filter-based filtering. After the nonlinear postfiltering, both the ringing and the blotchiness caused by remaining halftone noises are effectively reduced.

\section{Experimental Results}

We generated examples using a four-level DWT based on Johnston's 16-tap filters, although other filters may be used as well [11]. The $400 \times 400$ pel image shown in Fig. 3(a) is a $3 \times 6$ clustered dot halftone. The contone image was reconstructed by applying uniform- weight clipping and oriented filtering $(\alpha=1 / 3)$ to the subbands at three topmost levels. In addition, a combination of HMRF-based filter and $\sigma$ filter is used in postprocessing. The result is shown in Fig. 3(b). For illustration purposes, the four-level DWT of the halftone image is shown in Fig. 3(c). After clipping and oriented filtering, the subbands are shown in Fig. 3(d).

To demonstrate the robustness of the algorithm, we repeated the experiment for other types of halftones using the same parameters. An error diffused image is shown in Fig. 4(a), and its reconstructed contone version is shown in Fig. 4(b). Furthermore, the method can also be used for scanned material. We printed an image using $4 \times$ 8 cluster dots in a 300 dpi laser printer and scanned the printed material at 200 ppi. The scanned image is shown in Fig. 4(c), and its descreened version is shown in Fig. 4(d).

\section{EXTENDED Algorithm Using Overcomplete EXPANSION}

Other types of subband decompositions can also be applied to the descreening problem, particularly overcomplete representations, 

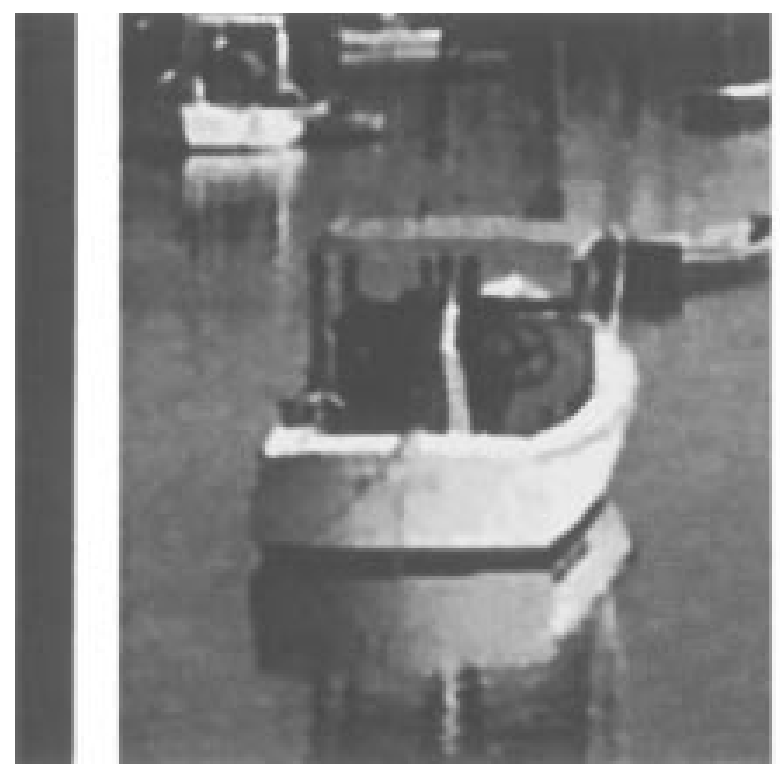

(a)
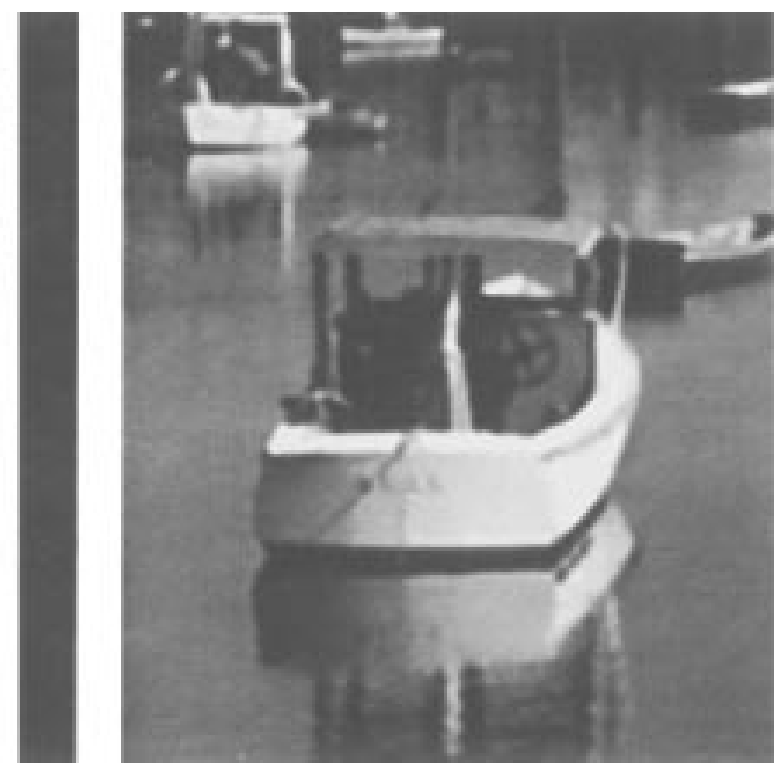

(b)

Fig. 5. (a) Reconstructed contone version of the clustered dot halftone image using algorithm II (overcomplete expansion). (b) Same for the error diffused image.
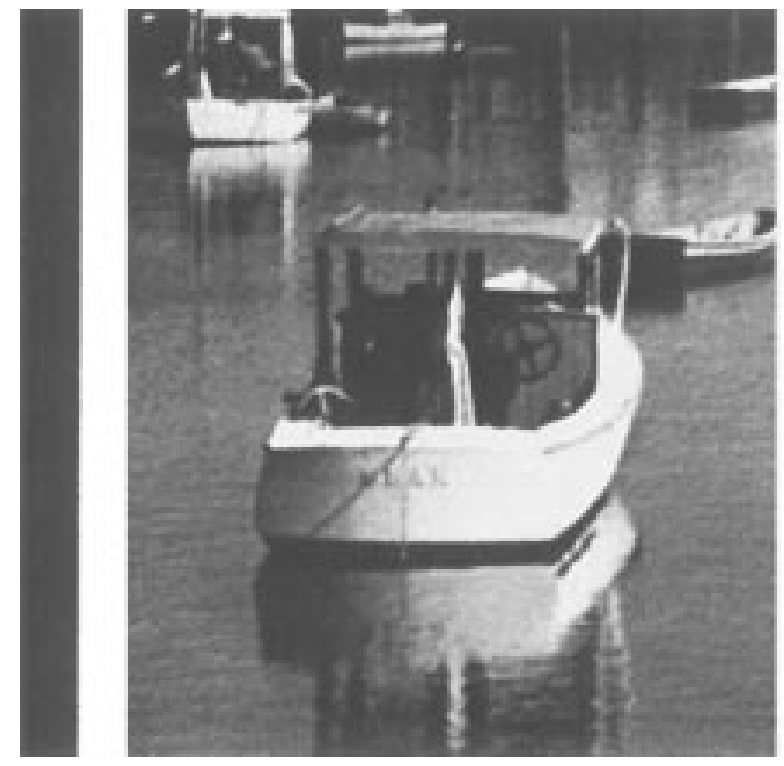

(a)
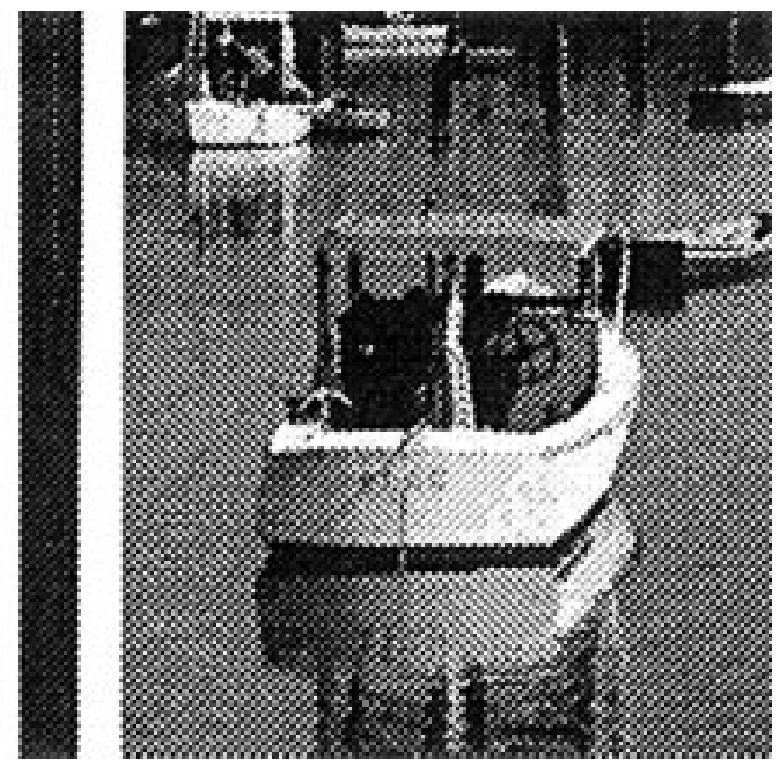

(b)

Fig. 6. Reconstructed contone version of the error diffused image using algorithm III (overcomplete expansion algorithm tuned for high-frequency halftones) is shown on the left. Use of this algorithm for lower-frequency halftones may produce incorrect results as shown on the right where it was applied to a clustered dot halftone.

in which filtered signals are not decimated during subband decomposition. One trivial restriction on the decomposition process is the invertibility of the decomposition. Our wavelet-based universal descreening method in Section II was improved by Xiong et al. in [15] by using the above-mentioned overcomplete representation instead of the critically decimated DWT. The process described in [15] is tailored to error diffusion halftoning, which does not present much low-frequency noise, and may fail for some common screening methods such as clustered dots. We propose an extension of our algorithm to include overcomplete decomposition using simple nondirectional nonlinear filters.

The halftone image $I$ can be preprocessed to eliminate spurious noise that do not contribute to the descreening process. Let $L_{0}=$
$F_{0}\{I\}$, where $F_{0}$ is an optional preprocessing filter. Hierarchical decomposition into lowpass and highpass bands is obtained as

$$
L_{k}=F_{k}\left\{L_{k-1}\right\} \quad H_{k}=L_{k-1}-L_{k}=L_{k-1}-F_{k}\left\{L_{k-1}\right\} .
$$

It is obvious that the image can be reconstructed by $L_{0}=$ $L_{N}+\sum_{i=1}^{N} H_{i}$. We process the highpass subband $H_{k}$ using noiseremoving filters and clipping to obtain $\hat{H}_{k}=G_{k}\left\{A\left\{H_{k}, \gamma_{k} H_{k-1}\right\}\right\}$, where

a) $A\{X, Y\}$ denotes the noise attenuation by clipping, comparing the parent subband $Y$ and the child subband $X$;

b) $\gamma_{k}$ is a scaling factor;

c) $G_{k}$ is an intraband filtering operator designed to remove halftone noise. 
TABLE II

PSNR PeRformances of DifFerent Algorithms for Image Lena

\begin{tabular}{l|c|c|c|c|c}
\hline & Algorithm I & Algorithm II & Algorithm III & Ref. [5] & Ref.[15] \\
\hline error diffusion & 30.8 & 29.4 & 31.7 & $32.0 / 31.0^{*}$ & 31.2 \\
\hline (3,6) clustered dot & 26.6 & 28.2 & 15.8 & N/A & $?$ \\
\hline
\end{tabular}

$\left(^{*}\right)$ - without using error diffusion weights information

The reconstructed contone image $R$ is obtained as

$$
R=L_{N}+\sum_{i=1}^{N} \hat{H}_{i}
$$

As an example, we can use uniform parameters across all levels. Let the intraband filter $G_{k}$ be a cascade of $2 \times 2$ averaging filter followed by a $5 \times 5 \sigma$ filter, where $\sigma=8$ seems to a be an adequate (but not necessarily optimal) choice. Let $\gamma_{k}=2$ and $F_{k}$ be the 7 $\times 7$ filter obtained by a double convolution of the $3 \times 3$ averaging filter. Nonlinear filtering such as the HMRF- or $\sigma$-based approaches can be applied at this stage to further clean the image and sharpen edges. In this case, the reconstructed contone versions of the halftone images shown in Fig. 3(a) and Fig. 4(a) are shown in Figs. 5(a) and (b), respectively.

Note that a compromise has to be made so that the algorithm works for a large number of halftones methods. If one wants to fine tune the algorithm to high-frequency halftones, one can set $N=1$ and use filter $F_{k}$ as a cascade of a $2 \times 2$ averaging filter and a $\sigma$ filter with $\sigma=32$ (which, again, is a good number but not necessarily optimal). Furthermore, we set $F_{0}$ as the $3 \times 3$ averaging filter and do not perform clipping. In this case, the reconstructed contone from the error-diffused image in Fig. 4(a) is shown in Fig. 6. The total complexity per processed pixel is two $2 \times 2$ and one $3 \times 3$ averaging filters, two $\sigma$ filters, and two additions.

Let us consider three algorithms:

I) the critically decimated decomposition from Section 2;

II) the overcomplete extension of I, described in this section;

III) the design of II for high-frequency halftones.

Algorithm I has lower complexity than II because the subbands are decimated. Hence, clipping, intraband filtering, and further wavelet stages are performed at lower resolution subband images. Algorithm III is simple but no longer universal.

Algorithm II does not show significant performance improvement over Algorithm I. In general, overcomplete decomposition will lead to sharper images but eliminates less halftoning noise. The same is true for Algorithm III. It leads to sharper images while retaining more halftone artifacts. Peak-signal-to-noise-ratio (PSNR) comparisons are shown in Table II for image Lena. Algorithm III achieves higher PSNR at a lower complexity than the one in [15]. However, it has the same limitations, i.e., it is not meant to work with lower frequency halftones, as shown in the example in Fig. 6.

\section{CONCLUSION}

We have presented a novel wavelet-based approach to recovering continuous tone images from halftone images. The main concept resides in the utilization of wavelet decomposition to represent the halftone image into a number of image subbands. The subbands are subject to intraband and interband operations designed to eliminate or attenuate unexpected patterns that are often indications of halftone noise presence. These operations come in the form of spatial and frequency selective processing that is intended to preserve most of the original image features while eliminating the halftone noise. Examples of features that are preserved through these selective operations are sharp and prominent edges. After the wavelet subbands are processed, they are used to reconstruct a contone image. This image can be optionally subject to further nonlinear filtering, as a postprocessing stage, which is intended to create the final aesthetic contone image. Both critically decimated and overcomplete subband decompositions were studied.

While Algorithm III is tuned to high-frequency halftones and requires low implementation complexity, Algorithm I works well across a large collection of halftoning methods. Furthermore, excellent contone images can be obtained from scanned halftone images. This approach is independent of parameter estimation and, therefore, can be viewed as practically universal to most types of halftoning techniques.

\section{REFERENCES}

[1] P. G. Roetling and R. P. Loce, "Digital halftoning," in Digital Image Processing Methods, E. Dougherty, Ed. New York: Marcel Dekker, 1994, ch. 10

[2] D. Kermisch and P. G. Roetling, "Fourier spectrum of halftone images," J. Opt. Soc. Amer., vol. 65, pp. 716-722, June 1975.

[3] M. Analoui and J. Allebach, "New results on reconstruction of continuous-tone from halftone," in Proc. IEEE Int. Conf. Acoust. Speech Signal Process., San Francisco, CA, 1992, vol. 3, pp. 313-316.

[4] Z. Fan, "Retrieval of images from digital halftones," in Proc. Int. Symp. Circuits Syst., May 1992, pp. 313-316.

[5] P. W. Wong, "Inverse halftoning and kernel estimation for errordiffusion," IEEE Trans. Image Processing, vol. 4, pp. 486-498, Aug. 1995.

[6] S. Hein and A. Zakhor, "Halftone to continuous-tone conversion of error-diffusion coded images," IEEE Trans. Image Processing, vol. 4, pp. 208-216, Mar. 1995.

[7] S. Schweitzer and R. Stevenson, "Bayesian approach to inverse halftoning," in Proc. SPIE Conf. Human Vision, Vision Process. Digital Display IV, 1993, pp. 282-292.

[8] Y. Kim, G. R. Arce, and N. Grabowski, "Inverse halftoning using binary permutation filters," IEEE Trans. Image Processing, vol. 4, pp. 1296-1311, Sept. 1995.

[9] M. Y. Ting and E. Riskin, "Error-diffused image compression using a halftone to gray scale decoder and predictive pruned tree-structured VQ," in Proc. SID, May 1993.

[10] Z. Fan, "Unscreening using a hybrid filtering approach," in Proc. IEEE Int. Conf. Image Process., 1996, vol. III, pp. 351-354.

[11] G. Strang and T. Nguyen, Wavelets and Filter Banks. Wellesley, MA: Wellesley-Cambridge, 1996.

[12] J. M. Shapiro, "Embedded image coding using zerotrees of wavelet coefficients," IEEE Trans. Signal Processing, vol. 41, pp. 3445-3462, Dec. 1993.

[13] J. Luo, C. W. Chen, and K. J. Parker, "On the application of Gibbs random field in image processing: From segmentation to enhancement," J. Electron. Imaging, vol. 2, no. 2, pp. 189-198, Apr. 1995.

[14] J. Lee, "Digital image smoothing and the $\sigma$-filter," Comput. Vis., Graph. Image Process., vol. 24, pp. 255-269, 1983.

[15] Z. Xiong, K. Ramchandran, and M. Orchard, "Inverse halftoning using wavelets," in Proc. Int. Conf. Image Process., Lausanne, Switzerland, 1996, vol. I, pp. 569-572. 